\title{
COMPETÊNCIA CULTURAL E QUALIDADE DA ASSISTÊNCIA DE ENFERMAGEM
}

\section{CULTURAL COMPETENCE AND QUALITY OF NURSING CARE}

\author{
Ana Luiza Ferreira Aydogdu ${ }^{*}$
}

\begin{abstract}
Resumo. Indivíduos migram por motivos muito variados, alguns estão em busca de melhores condições de trabalho e de vida, outros se veem obrigados a deixar seus países devido a guerras e conflitos. Além disso, o mercado do turismo de saúde está em ascensão, assim, pessoas também saem de suas regiões em busca de tratamentos médicos. A globalização facilitou os movimentos migratórios. Todos esses fatores fizeram com que a diversidade cultural dos povos fosse aumentando gradativamente. A cultura está diretamente ligada à assistência à saúde, pois interfere na forma como o indivíduo encara o processo saúde-doença. Por isso, profissionais de enfermagem devem conhecer as crenças e os costumes de seus pacientes para prestar um cuidado eficaz e eficiente. O objetivo deste estudo foi refletir sobre a enfermagem transcultural e a qualidade da assistência prestada pelos profissionais de enfermagem. Trata-se de uma revisão narrativa baseada na leitura, análise e interpretação de artigos científicos sobre enfermagem transcultural disponíveis na Biblioteca Virtual em Saúde (BVS) e no Google Acadêmico. Observou-se que as características culturais das populações de diversos países estão em constante processo de mudança. Cursos, intercâmbios e renovações curriculares buscam atualizar o estudante e o profissional de enfermagem sobre diferentes culturas, pois há o conhecimento de que os profissionais de enfermagem devem estar culturalmente capacitados para atender às necessidades de uma sociedade cada vez mais culturalmente diversificada. Conclui-se que faculdades de enfermagem e instituições de saúde devem atentar para o ensino e para a prática da enfermagem transcultural, para que a assistência de enfermagem possa ser mais qualificada e humanizada.
\end{abstract}

PALAVRAS-CHAVE: Assistência à Saúde. Diversidade Cultural. Enfermagem Transcultural. Enfermeiras e Enfermeiros.

Abstract. Individuals migrate for several different reasons, some looking for better working and living conditions, others are forced to leave their countries due to wars and conflicts. In addition, the health tourism market is on the rise, so people are also leaving their regions in search of medical care. Globalization has facilitated migratory movements. All these factors caused the cultural diversity of the nations to gradually increase. Culture is directly linked to health care, since it interferes with how individuals face the healthdisease process; therefore, nursing professionals must know the beliefs and behaviours of their patients to provide effective and efficient care. The aim of this study was to reflect on transcultural nursing and the quality of care provided by nursing professionals. It is a narrative review based on the reading, analysis, and interpretation of scientific articles on transcultural nursing available at the Virtual Health Library (VHL) and Google Scholar. It was observed that cultural characteristics of the populations of different countries are in a constant process of change. Courses, exchange programs and curricular changes seek to update the students and the nursing professionals about different cultures, as it is known that nursing professionals must be culturally competent to meet the needs of an increasingly culturally diverse society. It is concluded that nursing colleges and health institutions should pay attention to the teaching and practice of transcultural nursing, so that nursing care can be more qualified and humanized.

KEYWORDS: Health Care. Cultural Diversity. Transcultural Nursing. Nurses. 


\section{INTRODUÇÃO}

Pessoas migram por razões muito variadas, que podem ir desde buscas por melhores condições de trabalho e sociais, até fugas de territórios em guerra ou conflito. ${ }^{1}$ Avanços tecnológicos nas áreas do transporte e da comunicação facilitaram esses movimentos migratórios, que nos últimos anos estão cada vez mais comuns. ${ }^{2}$ Além disso, o avanço na área da saúde também aumentou o movimento de pessoas entre os países devido a um mercado cada vez mais popular, o turismo de saúde. ${ }^{2}$

A diversidade cultural dos povos tem uma relação direta com a prestação de cuidados, visto que a cultura interfere nas atitudes das pessoas frente à saúde e à doença. ${ }^{3}$ Para o profissional de enfermagem, reconhecer as diferenças culturais dos pacientes e saber como agir frente a crenças e hábitos diversificados é de fundamental importância para a prestação de uma assistência qualificada. ${ }^{4}$

Diferenças culturais podem interferir negativamente na interação enfermeiropaciente, podendo representar inclusive, riscos para a segurança tanto do profissional quanto do indivíduo que necessita de cuidados. 5,6 Assim, a enfermagem, uma profissão que está em constante processo de aprendizagem, tendo que se adaptar

\section{RESULTADOS E DISCUSSÃO}

Enfermeiros e docentes de enfermagem em diversos países ao redor do mundo atentam para a importância da enfermagem transcultural. 4,5,16,25 Para que o profissional de enfermagem possa oferecer um cuidado de qualidade aos pacientes de ao surgimento de novas doenças, novos tratamentos e novos cuidados 7 foi, através dos tempos, se adaptando também às diferenças culturais dos pacientes e de seus familiares. 4

Por volta da década de 50, Leininger, a pioneira da enfermagem transcultural, alertou para importância de incorporar disciplinas como antropologia, sociologia e psicologia às práticas de enfermagem, visando assim aumentar a eficácia e a eficiência dos cuidados prestados pelos profissionais da área. ${ }^{8}$ Enfermagem transcultural é, portanto, a prática da enfermagem baseada nas características culturais do paciente visando manter ou promover sua saúde física, mental e social. ${ }^{9}$

No Brasil, um país historicamente reconhecido por sua diversidade cultural, a importância da enfermagem transcultural é evidente. ${ }^{10}$ Além disso, o país vem aumentando seu potencial para desenvolver o turismo em saúde, ${ }^{11-13}$ o que faz com que o profissional de enfermagem deva estar cada vez mais preparado para atender às necessidades de indivíduos de diferentes etnias. O objetivo deste estudo foi fazer uma reflexão sobre a enfermagem transcultural e a qualidade da assistência prestada pelo profissional de enfermagem. diferentes origens culturais é fundamental que ele seja culturalmente competente, ${ }^{4}$ ou seja, é preciso ter sensibilidade, conhecimentos e habilidades para atender indivíduos com características culturais diferentes. Diferenças estas que vão desde crenças e costumes até 
o próprio idioma falado pelo paciente e seus familiares; ${ }^{16-18,21,25}$ estes fatores culturais podem representar verdadeiras barreiras entre o profissional de enfermagem e o paciente.

Faculdades de enfermagem e instituições de saúde de diferentes países, percebendo mudanças constantes nas características das populações que atendem, procuram tornar estudantes e profissionais de enfermagem cada vez mais competentes na prática da enfermagem transcultural através de intercâmbios, ${ }^{19}$ cursos $^{4}$ e avaliações curriculares. ${ }^{20,22}$ Ainda assim, as alterações demográficas de algumas regiões ocorrem tão rapidamente, que é difícil acompanhar. Alguns países, como os Estados Unidos da América (EUA), ${ }^{26}$ Canadá $^{27}$ e Brasil, ${ }^{10}$ devido a própria formação histórica têm uma diversidade cultural muito grande. Outros como Turquia e Jordânia tiveram seus cenários demográficos ainda mais alterados devido a chegada de refugiados. ${ }^{28}$ Países como Arábia Saudita, Alemanha, Rússia, Reino Unido e os Emirados Árabes Unidos, têm muitos imigrantes; ${ }^{28}$ e países asiáticos como Malásia, Singapura e Índia são importantes destinos para o turismo de saúde. ${ }^{29}$ Todos estes fatores fazem com que profissionais de enfermagem que desempenham suas funções nesses países e em outros com atributos semelhantes, prestem cuidados a indivíduos com características culturais muito diversificadas, o que pode ser um verdadeiro desafio.

A importância da prática da enfermagem transcultural se faz presente, principalmente, devido aos riscos que podem ser gerados em consequência de desentendimentos originados pela existência de hábitos, crenças e idiomas diferentes.
Interferências na comunicação, entre o paciente e o profissional de enfermagem, podem acarretar erros que impedem tanto a prevenção de doenças, quanto a promoção da saúde. ${ }^{5,6}$ Para que o profissional de enfermagem possa oferecer um cuidado de enfermagem holístico à sociedade, é preciso que ele conheça bem a população que atende, sendo fundamental entender e respeitar as diferentes culturas desses indivíduos.

Num estudo feito na Turquia, país que atualmente recebe o maior de número de refugiados no mundo, ${ }^{28}$ foram identificadas atitudes negativas de estudantes de enfermagem ao prestarem cuidados aos refugiados Sírios. ${ }^{17} \mathrm{Na}$ Jordânia, um outro país com um número elevado de refugiados, ${ }^{28}$ num estudo realizado com estudantes de enfermagem, que atendiam a essa população, foi observada a necessidade de vencer a barreira da língua estrangeira e preparar o discente de enfermagem tanto na teoria, como na prática para prestar assistência a pacientes de origens culturais diferentes. ${ }^{21}$ Já um estudo feito no Canadá, um país culturalmente diversificado, atentou para a necessidade de avaliar os currículos das faculdades de enfermagem com relação ao ensino da enfermagem transcultural; ${ }^{22}$ e um estudo feito em outro país cujo cenário demográfico é diversificado, os EUA, identificou um aumento da eficiência cultural de estudantes de enfermagem após um curso de enfermagem transcultural. ${ }^{4}$

Um estudo feito com enfermeiras trabalhando na Arábia Saudita e nos Emirados Árabes Unidos, países com grande número de imigrantes, ${ }^{28}$ enfatizou a necessidade de práticas educacionais focadas na cultura e nas crenças religiosas para que profissionais de enfermagem ganhem competência cultural. ${ }^{16}$ 
Tabus culturais e língua estrangeira foram apontados como desafios para a enfermagem em um estudo realizado em Singapura, ${ }^{25}$ um país que recebe muitos turistas em busca de tratamento médico. ${ }^{29} \mathrm{Em}$ outro país reconhecido como destino para o turismo de saúde, a Índia, ${ }^{29}$ um estudo feito com enfermeiros apontou que um conhecimento mais profundo da própria cultura faz com que o indivíduo respeite e tome atitudes mais conscientes com relação aos hábitos culturais dos outros, facilitando assim a interação enfermeiro-paciente. ${ }^{6}$ Estudos feitos com estudantes e profissionais de enfermagem em diferentes países mostram a existência de um nível alto ${ }^{17,18}$ ou moderado ${ }^{24}$ de sensibilidade intercultural, mas ainda assim existe uma falta de conhecimento sobre a enfermagem transcultural, ${ }^{17,18}$ pouca autoconfiança ao cuidar de pacientes de outras culturas ${ }^{24}$ e falta de interesse em aprender sobre o tema. ${ }^{18}$

No cenário nacional contamos com índios, quilombolas, ciganos e imigrantes entre outros grupos, cujas características culturais são distintas. Além disso, grupos formados por indivíduos com diferentes orientações sexuais também têm características culturais diferenciadas. ${ }^{10}$ Um outro fator que deve ser considerado é que o Brasil é um país com potencial para se desenvolver em áreas do turismo de saúde, como o turismo de bemestar $^{12}$ e o turismo médico, ${ }^{11,13}$ precisando, portanto, estar preparado para atender indivíduos de diferentes origens culturais.

\section{CONSIDERAÇÕES FINAIS}

Através da presente reflexão, identificou-se que o perfil populacional está sofrendo alterações em diversos países, e que a competência cultural é de fundamental importância para que os profissionais de
Estudos feitos no âmbito nacional mostram que ainda existe uma falta de conhecimento com relação aos direitos da população LGBTQIA+ e que a prestação de cuidados de enfermagem a estes indivíduos precisa ser mais eficiente e humanizada. ${ }^{30-32}$ Outro estudo feito no Brasil enfatizou a importância do intercâmbio para a prática de enfermagem, através do qual o enfermeiro alcança não somente $o$ crescimento profissional, mas também pessoal, entretanto, neste mesmo estudo o idioma estrangeiro foi apresentado como um grande desafio a ser vencido. ${ }^{19} \mathrm{Um}$ estudo feito na região centro-oeste do Brasil apontou a falta de uma disciplina no currículo de enfermagem voltada para as populações tradicionais que habitam a região, como indígenas e quilombolas. ${ }^{20}$ Outro estudo realizado no país, junto aos quilombolas, enfatizou a importância da enfermagem transcultural na prevenção de doenças e promoção da saúde das minorias populacionais. ${ }^{23}$

Avanços trazidos pela globalização fizeram com que o movimento de indivíduos entre os países crescesse rapidamente, esse processo provavelmente continuará aumentando através dos anos, mudando cada vez mais o cenário cultural das regiões, com isso mudam também as necessidades individuais e os perfis de saúde e doença da população. O profissional de enfermagem deve estar preparado para atender essa sociedade cada vez mais diversificada.

enfermagem possam cumprir com suas funções, prestando um cuidado eficiente e humanizado à sociedade e respeitando as diferenças culturais das pessoas. Portanto, faculdades de enfermagem do mundo inteiro, 
incluindo as do Brasil, devem acompanhar essas mudanças estando preparadas para formar profissionais culturalmente competentes, atentando ainda para a necessidade do conhecimento de idiomas estrangeiros por parte do profissional de enfermagem. Além disso, cursos de educação continuada devem contar com temas sobre enfermagem transcultural, garantindo assim

\section{REFERÊNCIAS BIBLIOGRÁFICAS}

1. Lise F, Schwartz E, Krumwiede N, BarbieriFigueiredo MC. Enfermagem no cuidado às famílias migrantes e refugiadas: tema emergente. J. nurs. health. 2019; 9(1): e199111.

2. Ferreira KL, Schreiber D, Puffal DP. Análise reflexiva do turismo em saúde à luz da inovação. Revista Brasileira de Pesquisa em Turismo. 2016; 10(2): 25473.

3. Langdon EJ, Wiik FB. Anthropology, health and illness: an introduction to the concept of culture applied to the health sciences. Rev. Latino-Am. Enfermagem. 2010; 18(3): 459-66.

4. James L, Stiles A, Stephens C. Nursing student differences in transcultural self-efficacy by culture curriculum: a longitudinal study. J Transcult Nurs. 2020; 25: 1043659620950422.

5. Campelo CL, Sousa SMA, Silva LDC, Dias RS, Azevedo PR, Nunes FDO et al. Cultura de segurança do paciente e cuidado cultural de enfermagem. REUOL. 2018; 12(9): 2500-06.

6. Larsen R, Mangrio E, Persson K. Interpersonal communication in transcultural nursing care in India: a descriptive qualitative study. J. Transcult. Nurs. 2020; 00(0):1-8.

7. Qalehsari MQ, Khaghanizadeh M, Ebadi A. Lifelong learning strategies in nursing: a systematic review. Electro. Physician. 2017; 9(10): 5541-50. que pacientes de diferentes culturas possam receber um cuidado de enfermagem eficaz e eficiente.

Espera-se com esse estudo incentivar pesquisas que utilizem diferentes desenhos metodológicos para abordar temas relativos à enfermagem transcultural e à competência cultural dos profissionais de enfermagem que atuam no âmbito nacional.

8. Queiroz MVO, Pagliuca LMF. Conceito de enfermagem transcultural: análise de seu desenvolvimento em uma dissertação de mestrado. R. Bras. Enferm. 2001; 54(4): 630-37.

9. Leininger $M$. Nursing education exchanges: concerns and benefits. J. Transcult. Nurs. 1998; 9(2): 57-63.

10. Leal S. Diversidade Cultural e Reconhecimento no Quadro de Políticas de Comunicação e Cultura no Brasil: desafios e perspectivas para concretização da Convenção da Unesco de 2005. Polít. soc. 2017; 16(35): 94-131.

11. Frozé VD, Gianotti HPP, Gianotti PSP. Considerações sobre o turismo de saúde na América Latina - serviços de primeiro mundo com preços de países emergentes? Revista Hospitalidade. 2010; VII(1):32-42.

12. Machado ALM, Pinent MAP. O turismo de bemestar como possibilidade de desenvolvimento de um cluster turístico: a proposta do Vale Do Paranhana Rio Grande do Sul. Agora. 2017; 19(2): 54-66.

13. Ribeiro HC, Baptista MLC. Porto Alegre como cenário de turismo de saúde. Temas Educ. Saúde. 2019; 15(2): 291-12.

14. Mendes-Da-Silva W. Contribuições e limitações de revisões narrativas e revisões sistemáticas na área de 
negócios. Rev. Adm. Comtemp. 2019; 23(2): 1-11.

15. Rother ET. Systematic literature review X narrative review. Acta Paul. Enferm. 2007; 20(2): 5-6.

16. Al-Yateem N, Alyateem S, Rossiter R. Cultural and religious educational needs of overseas nurses working in the Kingdom of Saudi Arabia and United Arab Emirates. Holist. Nurs. Pract. 2015; 29(4):20515.

17. Alici NK. Cultural sensitivity and attitudes towards refugees of Turkish nursing students: a cross sectional descriptive study. Int. J. Intercult. Relat. 2021; 80:1-6.

18. Arli SK, Bakan AB. Cerrahi hemsirelik merhamet ve kulturlerarasi duyarliligi etkileyen faktorler (The factors affecting compassion and intercultural sensitivity among the surgical nurses). STED. 2018; 27(4): $277-83$.

19. Bardaquim VA, Dias EG. A realização de intercâmbio no doutorado em enfermagem: um relato de experiência. J. nurs. health. 2019; 9(1): e199104.

20. Castro NJC, Mesquit DS, Naka KS, Teixeira JBG, Borges RS. Ensino da saúde das populações tradicionais em cursos de enfermagem. Enferm. Foco. 2019; 10(6): 36-41.

21. Dotevall C, Winberg E, Rosengren K. Nursing students' experiences with refugees with mental health problems in Jordan: a qualitative content analysis, Nurse Educ. Today. 2018; 61:155-61.

22. Rowan MS, Rukholm E, Bourque-Bearskin L, Baker C, Voyageur E, Robitaille A. Cultural competence and cultural safety in Canadian Schools of Nursing: a mixed methods study. Int. J. Nurs. Educ. Scholarsh. 2013; 10(1): 1-10.

23. Santos ANS, Nascimento ER. Proposições de cuidado cultural à enfermagem frente a aspectos da saúde reprodutiva de mulheres quilombolas. Rev. baiana enferm. 2019; 33:e33375.
24. Toda T, Maru M. Cultural sensitivity of Japanese nurses: exploring clinical application of the intercultural sensitivity scale. Open J. Nurs. 2018; 8: 640-55.

25. Tay LH, Ang E, Hegney D. Nurses' perceptions of the barriers in effective communication with inpatient cancer adults in Singapore. J. Clin. Nurs. 2012; 21(17-18): 2647-58.

26. Eller JD. Culture and diversity in the United States: so many ways to be American. $1^{\text {a }}$ ed. London: Routledge; 2015.

27. Bohatyrets V. Embracing multiculturalism of Canada: the roots \& the present-day realities. Modern Historical and Political Issues: Journal in Historical \& Political Sciences. - Chernivtsi National University [Internet]. 2017; 35-36: 32-41.

28. United Nations. Department of Economic and Social Affairs Population Division. The world counted 258 million international migrants in 2017, representing 3.4 percent of global population. Population Facts [Internet]. 2017 [cited $2021 \mathrm{Apr}$ 13]; 5:1-5. Available from: https://www.un.org/en/ development/desa/population/publications/pdf/ popfacts/PopFacts_2017-5.pdf

29. Kim S, Arcodia C, Kim I. Critical success factors of medical tourism: the case of South Korea. Int. J. Environ. Res. Public Health. 2019; 16(24): 4964.

30. Fernandes MCL, da Silva W, Tolentino TS, Araújo MJA, Joventino MLS, Silva PE. Conhecimento de profissionais de enfermagem acerca da assistência à saúde dos transexuais. Rev. de Ciênc. Saúde Nova Esperança. 2019; 17(2): 34-44.

31. Silva DD, Silva FAB, Sobreira MVS, Andrade ALF, Araújo EB, Araújo AF. Assistência de enfermagem na atenção básica a população homossexual da cidade de Caicó-RN. Temas em Saúde. 2018; 18(3): 271-96.

32. Laporte NLO, Assis MA. Conhecimentos dos graduandos de enfermagem voltados para a assistência dos indivíduos LGBTQIA+. Revista Científica UMC. 2020; 5(2). 